\title{
LncRNA PRNCR1 promotes breast cancer proliferation and inhibits apoptosis by modulating microRNA-377/CCND2/MEK/MAPK axis
}

Jian Ouyang

Pingxiang Health Vocational College

\section{Zilong Liu}

Pingxiang Health Vocational College

\section{Xiaobing Yuan}

Pingxiang Health Vocational College

\section{Xia Chen}

Pingxiang Health Vocational College

\section{Yongpeng Wang}

Pingxiang Health Vocational College

Lu Liu

Pingxiang Health Vocational College

\section{Chunping Long}

Pingxiang Health Vocational College

Hui Liang ( $\sim$ huiliang120903@163.com )

Pingxiang Health Vocational College https://orcid.org/0000-0001-8786-2794

\section{Research}

Keywords: LncRNA PRNCR1; Breast cancer; microRNA-377; CCND2; MEK/MAPK pathway; Proliferation; Apoptosis

Posted Date: March 30th, 2020

DOI: https://doi.org/10.21203/rs.3.rs-19525/v1

License: (c) (i) This work is licensed under a Creative Commons Attribution 4.0 International License. Read Full License

Version of Record: A version of this preprint was published at Archives of Medical Research on July 1st, 2021. See the published version at https://doi.org/10.1016/j.arcmed.2021.01.007. 


\section{Abstract}

Background: Long non-coding RNAs (IncRNAs) have recently become the vital gene regulators in diverse cancers. In our study, we purposed to inquiry into the mechanisms of IncRNA PRNCR1 in breast cancer via microRNA-377 (miR-377)/CCND2/MEK/MAPK axis.

Methods: PRNCR1 expression in breast cancer tissues was detected, and the correlation between PRNCR1 expression and prognostic survival was analyzed. The expressions of PRNCR1 and miR-377 in breast cancer cell lines were detected. Relationships among PRNCR1, miR-377 and CCND2 were confirmed by luciferase activity, RNA pull-down or RIP assays. Breast cancer cells were introduced with silenced PRNCR1 or restored miR-377 to explore their functions in breast cancer's malignant phenotype. Related proteins expression in MEK/MAPK pathway was determined by western blot analysis.

Results: PRNCR1 was abnormally highly expressed and miR-377 was poorly expressed in breast cancer, and patients with high expression of PRNCR1 had a poor prognosis. PRNCR1 silencing or miR-377 overexpression resulted in suppressed breast cancer cell proliferation ability, blocked cell cycle process and induced apoptosis. PRNCR1 may participate in the modulation of CCND2 by competitively binding with miR-377. CCND2 activated the MEK/MAPK pathway, and after treatment with Mirdametinib, the MEK/MAPK pathway was inhibited, which was found to retard breast cancer growth.

Conclusion: Our study highlights that IncRNA PRNCR1 may competitively bind to miR-377, leading to upregulated CCND2, which in turn leads to MEK/MAPK pathway activation, thereby promoting breast cancer growth.

\section{Introduction}

Breast cancer is a heterogeneous malignancy that contributes to uncontrolled propagation and diffusion of breast tissue cells [1,2]. In recent years, the mortality of breast cancer has been decreased in most high-income countries thanks to the developed therapy and earlier diagnosis., while an enhanced mortality still exists in certain countries [3]. Breast cancer is mainly caused by gender, age, alcohol consumption, oral contraceptive, hereditary tendency and family history [4]. Great achievements have been made in the diagnostic and therapeutic methods for breast cancer, resulting in the decrease of mortality rate. Nevertheless, over 500,000 deaths have been found each year around the world [5]. Surgical resection, chemotherapy and radiotherapy are vital treatment regimens for breast cancer, but these methods are not suitable for advanced metastatic breast cancer [6]. Therefore, more efficient therapeutic strategies of breast cancer are needed to further investigate the functions associated with tumor growth and development of breast cancer.

Long non-coding RNAs (IncRNAs) have been affirmed in diverse cancers and exert critical functions through interacting with DNA, RNA, or protein molecules [7]. LncRNA prostate cancer non-coding RNA 1 (PRNCR1) is a $13 \mathrm{~kb}$ intron-less IncRNA, and it is transcribed from the 8q24 'gene-desert' region [8]. LncRNA PRNCR1 is a newly gene to be an oncogene in prostate cancer [9], colorectal cancer [10], as well 
as gastric cancer [11]. It is reported that IncRNA PRNCR1-2 takes part in breast cancer cells [12]. MicroRNAs (miRNAs) post-transcriptionally modulate gene expression via targeting the 3'untranslated region (3'UTR) of mRNAs [13]. MiRNAs are being underlined for their roles in pathological processes, including tumor formation and development in breast cancer $[14,15]$. The microRNA miR-377 is located in the 14q32.31 locus, and this locus harbors genes that coding for a large number of miRNAs involved in controlling biological functions in metastatic cancer cells [16]. Evidence has shown that miR-377-3p plays a role in triple-negative breast cancer cell lines [17]. CyclinD2 (CCND2) is a unique gene among the three D-type cyclins, which is up-regulated in the growth arrest in normal fibroblasts [18]. An article has demonstrated that upregulated CCND2 increases proliferation in breast cancer cells [19]. Based on aforementioned evidence, we could conjecture that IncRNA PRNCR1/miR-377/CCND2 axis participates in breast cancer growth.

\section{Materials And Methods}

\section{Clinical sample collection}

From February 2013 to June 2014, 64 patient samples from breast cancer resections along with the corresponding paracancerous tissues were collected from Pingxiang Health Vocational College. The pathological diagnosis was obtained based on histology or biopsy of the tumor specimen and examined by experienced pathologists. The breast cancer tissues and paracancerous tissues were stored in liquid nitrogen.

\section{Cell culture and transfection}

Human breast epithelial cell line (MCF-10A) and breast cancer cell lines (MDA-MB-231, MCF-7, BT-549, MDA-MB-468 and SK-BR-3) without mycoplasma and other contamination were selected. The above cells were purchased from American Type Culture Collection (Manassas, VA, USA). All cells were cultured in 90\% RPMI-1640 medium, which were appended with 10\% fetal bovine serum (Gibco; Thermo Fisher Scientific, Waltham, MA, USA), $100 \mathrm{U} / \mathrm{mL}$ penicillin and $100 \mathrm{mg} / \mathrm{mL}$ streptomycin, and then cultured in a saturated incubator containing $5 \% \mathrm{CO}_{2}$ at $37^{\circ} \mathrm{C}$.

MCF-7 cells were divided into groups, and the vector used for transfection was purchased from Guangzhou RiboBio (Guangdong, China). siRNA was designed and synthesized by Thermo Fisher Scientific Inc. MCF-7 cells were seeded at $1 \times 10^{6}$ cells/well in 6-well plates, and transduced with Lipofectamine 2000 transfection reagent (Invitrogen, Carlsbad, CA). According to different experimental requirements, cells were collected at different time periods after transfection for subsequent experiments.

\section{Reverse transcription quantitative polymerase chain reaction (RT-qPCR)}

Cells were harvested $48 \mathrm{~h}$ post transfection and total RNA was extracted using Trizol (Sigma-Aldrich, St Louis, MO, USA). RNA sample $(5 \mu \mathrm{L})$ was diluted 20 -fold with RNase-free ultrapure water, and the 
absorbance value at $260 \mathrm{~nm}$ and $280 \mathrm{~nm}$ was read by an ultraviolet spectrophotometer to determine the concentration and purity of RNA. According to the reverse transcription kit instructions (Beyotime, Shanghai, China), reverse transcription was performed on a PCR amplification instrument for the synthesis of a cDNA template. The required qPCR primers were synthesized by Sangon Biotech Co., Ltd. (Shanghai, China). The primer information is shown in Table 1. Glyceraldehyde phosphate dehydrogenase (GAPDH) was the loading control of PRNCR1 and CCND2, and U6, the loading control of miR-377. $2^{-\triangle \Delta C t}$ method was adopted for gene expression analysis.

Table 1

Primer sequence

\begin{tabular}{|ll|}
\hline Name & Sequence (5'-3') \\
\hline PRNCR1 & F: CCAGATTCCAAGGGCTGATA \\
\hline miR-377 & F: GATGTTTGGAGGCATCTGG \\
\hline CCND2 & R: GGCATCACACAAAGGCAAC \\
\hline F : ACCTTCCGCAGTGCTCCTA \\
\hline R: CCCAGCCAAGAAACGGTCC \\
\hline FAPDH & R: GAAGCAAATCATCGGACGACC \\
\hline $\begin{array}{l}\text { Note: F, forward; R: reverse; miR-377, microRNA-377; GAPDH, glyceraldehyde phosphate } \\
\text { dehydrogenase. }\end{array}$ & R: AGTAGAGGCAGTGTTTCCTCGG \\
\hline
\end{tabular}

\section{Western blot assay}

Cells were harvested $48 \mathrm{~h}$ post transfection and then washed with precooled phosphate buffered saline (PBS) and lysed with radioimmunoprecipitation assay buffer containing $10 \%$ protease inhibitor (MCE, USA). The cell sample was transferred to a $1.5 \mathrm{~mL}$ centrifuge tube, centrifuged for $10 \mathrm{~min}$ at $13,000 \mathrm{~g}$ for acquiring the supernatant. The measurement of protein concentration was implemented by bicinchoninic acid method and stored at $-20^{\circ} \mathrm{C}$ until use. The kit of sodium dodecyl sulfate polyacrylamide gel electrophoresis was utilized for preparing $10 \%$ separation gel and $4 \%$ concentrated gel. Next, the protein was separated by electrophoresis on polyacrylamide gel, and transferred to the nitrocellulose membrane by wet transfer method, and the membrane was blocked by $5 \%$ defatted milk for $1 \mathrm{~h}$. After that, the membrane was probed with the primary antibodies CCND2 (ab207604), MEK1 (ab32091), phosphorylated (p)-MEK1(ab96379), p38 MAPK (ab31828), p-p38 MAPK (ab4822) (all from Abcam, Cambridge, USA), and re-probed with secondary antibody IgG (ab150077, Abcam). The image was 
developed by a Bio-Rad gel imaging system (MG8600, Beijing Thmorgan Biotechnology Co., Ltd., Beijing, China), and quantitative analysis was performed using IPP7.0 software (Media Cybernetics, Singapore).

\section{Cell counting kit (CCK)-8 assay}

According to the kit's operating instructions, CCK-8 (Bimake) was adopted to determine cell proliferation capacity. Twenty-four hours post cell transfection, the cells were re-seeded at 3000 cells per well onto a 96-well plate. Optical density value was detected using a microplate reader at $450 \mathrm{~nm}$. Each experiment was executed in triplicate and repeated independently three times

\section{Flow cytometry}

The cells at $48 \mathrm{~h}$ post transfection were rinsed with PBS-balanced salt solution, and detached with $0.25 \%$ trypsin. The trypsin was removed when the cells were observed to be shrinkage round under the microscope, and the detachment was terminated by adding serum-containing medium. Afterwards, the cells were triturated for cell suspension, which was centrifuged to remove the supernatant. Afterwards, the cells were fixed with $70 \%$ ice-cold ethanol for $30 \mathrm{~min}$, and dyed with $1 \%$ propidium iodide $(\mathrm{PI})$ solution containing RNA enzyme for $30 \mathrm{~min}$. With PI removal, the cells were adjusted to the concentration of $1 \mathrm{~mL}$. The samples were positioned in a BD-Aria FACS Calibur flow cytometer (FACSCalibur, Beckman Coulter, USA) for detecting the cell cycle.

The cells at $48 \mathrm{~h}$ post transfection were altered to the concentration of $1 \times 10^{6} \mathrm{cells} / \mathrm{mL}$. Next, the cells were appended with $70 \%$ precooled ethanol solution and rinsed with PBS two times. Cell suspension (100 $\mu \mathrm{L}$, no less than $10^{6}$ cells $/ \mathrm{mL}$ ) was suspended in $100 \mu \mathrm{L}$ Binding Buffer, supplemented with added with $10 \mu \mathrm{L}$ Annexin V-fluorescein isothiocyanate (FITC), $5 \mu \mathrm{L} \mathrm{PI}$ as well as $300 \mu \mathrm{L}$ Binding Buffer. A flow cytometer (Attune NxT, Thermo Fisher, USA) was implemented to detect apoptosis at $488 \mathrm{~nm}$.

\section{Fluorescent in Situ Hybridization (FISH)}

PRNCR1 expression was detected in situ in MCF-7 cells using a FISH Kit (C10910, RiboBio). The cell slide was placed on the 24-well plate's bottom, and MCF-7 cells were detached onto the slide (approximately 6 $\times 10^{4} /$ well). When reaching $60-70 \%$ confluence, cells were fastened in $4 \%$ paraformaldehyde, appended with $1 \mathrm{~mL}$ pre-cooled permeable fluid. After discarding the permeable fluid, cells were supplemented with $200 \mu \mathrm{L}$ prehybridization solution. Meanwhile, the hybridization solution was added with $2.5 \mu \mathrm{L} 20 \mu \mathrm{M}$ FISH Probe Mix storage fluid. Afterwards, the pre-hybridization solution was removed and hybridization solution containing the PRNCR1 probe was appended. Next, the cells were washed with different kinds of wash solution and incubated devoid of light. The nucleus was stained with ',6-diamidino-2-phenylindole $2 \mathrm{hci}$ solution and washed with PBS three times. Under dark conditions, the cell slide was carefully removed from the well, which was fixed on a glass slide with a mounting plate and perform fluorescence detection. PRNCR1 specific probe was synthesized by RiboBio.

\section{Dual luciferase reporter gene assay}


The biological prediction website was utilized to analyze the binding site of PRNCR1 and miR-377, and the binding site of miR-377 and CCND2, thereby obtaining the fragment sequence containing the action site. Next, the full length PRNCR1 and 3'UTR of CCND2 were cloned and amplified into pmirGLO luciferase vectors (E1330, Promega, Madison, WI, USA) and named as PRNCR1-wild type (Wt) and CCND2-Wt. Bioinformatics software was adopted for forecasting the binding sites of miR-377 and PRNCR1, along with miR-377 and CCND2 by site-directed mutations. PRNCR1-Mut and CCND2-mutant type (Mut) vectors were constructed, and the internal reference was pRL-TK vector (E2241, Promega) expressing renilla luciferase. The 293T cells were introduced with miR-377 mimic or mimic negative control (NC) with the luciferase reporter vector respectively, and the fluorescence intensity was tested using a fluorescence detector (Glomax 20/20, Promega).

\section{RNA pull-down assay}

Cells were transduced with $50 \mathrm{nM}$ biotin-labeled Wt-bio-miR-377 and Mut-bio-miR-377. Forty-eight hours later, cells were incubated for 10 min with specific lysis buffer (Ambion, Austin, Texas, USA). After that, the lysate was cultured with M-280 streptavidin magnetic beads (S3762, Sigma) pre-coated with RNasefree bovine serum albumin together with yeast tRNA (TRNABAK-RO, Sigma). Next, the beads were cultured for $3 \mathrm{~h}$ at $4{ }^{\circ} \mathrm{C}$, washed with pre-chilled lysis buffer, low salt buffer, and high salt buffer. The bound RNA was purified by Trizol and then detected.

\section{Radioimmunoprecipitation (RIP) assay}

Cell were lysed with lysis buffer ( $25 \mathrm{mM}$ Tris-HCl pH 7.4, $150 \mathrm{mM} \mathrm{NaCl}, 0.5 \%$ NP-40, 2 mM EDTA, 1 mM $\mathrm{NaF}$ and $0.5 \mathrm{mM}$ dithiothreitol) containing RNasin (Takara, Dalian, China) and a protease inhibitor mixture (B14001a, Roche, USA). The lysate was centrifuged to collect the supernatant. Then, anti-Ago-2 magnetic beads (BMFA-1, Biomarker, Beijing, China) were added, and the control group was added with anti-IgG magnetic beads. Then the beads were rinsed three times with wash buffer $(50 \mathrm{mM}$ Tris- $\mathrm{HCl}$, $300 \mathrm{mM} \mathrm{NaCl} \mathrm{pH} \mathrm{7.4,} 1 \mathrm{mM} \mathrm{MgCl}$ 2, 0.1\% NP-40). RNA was extracted from magnetic beads by Trizol method.

\section{Statistical analysis}

All data were processed by SPSS 21.0 statistical software (IBM Corp. Armonk, NY, USA). All data were reported as mean \pm standard deviation for no less than three independent experiments. The statistical significance of the average was determined by the student $t$ test. For skewed distribution data, nonparametric tests were used to determine statistical significance. $P<0.05$ was indicative of statistical significance.

\section{Results}

\section{LncRNA PRNCR1 is enhanced in breast cancer, and downregulated PRNCR retards breast cancer growth}


First, we collected 64 samples of breast cancer and paracancerous tissues for detecting PRNCR1 expression by RT-qPCR. As indicated in Fig. 1A, we discovered that PRNCR was elevated in breast cancer tissues. Patients were followed up after surgery to collect the prognostic survival time. At the same time, the breast cancer tissues were divided into PRNCR1 high expression group $(n=32)$ and PRNCR1 low expression group $(\mathrm{n}=32)$ based upon the median PRNCR1 expression, and the result of which indicated that high expression of PRNCR1 had poor prognostic survival (Fig. 1B). Human breast epithelial cell line MCF-10A and breast cancer cell lines MDA-MB-231, MCF-7, BT-549, MDA-MB-468 and SK-BR-3 were obtained for detecting PRNCR1 expression in cells (Fig. 1C). The findings indicated that PRNCR1 expression was increased to varying degrees in breast cancer cell lines in comparison to MCF-10A cell line. Therefore, MCF-7 cells with the highest PRNCR1 expression were selected for further assays.

Additionally, MCF-7 cells were transfected with si-NC and si-PRNCR1 to verify the transfection efficiency by RT-qPCR (Fig. 1D). The outcomes suggested that MCF-7 cells upon si-PRNCR1 treatment exhibited reduced viability, retarded cell cycle progression and induced proportion of apoptosis (Fig. 1E-G). The above results show that PRNCR1 can promote breast cancer cell growth.

\section{miR-377 competitively binds with IncRNA PRNCR1}

Subsequently, we detected the localization of IncRNA PRNCR1 in MCF-7 cells by RNA-FISH, and the results showed that it was mainly localized in the cytoplasm (Fig. 2A). The online website predicted that a binding site existed between PRNCR1 and miR-377 (Fig. 2B). The dual luciferase reporter gene assay verified that miR-377 overexpression inhibited the activity of the PRNCR1 fluorescent reporter vector (Fig. 2C). RNA pull-down assay indicated that PRNCR1 bound by Wt-miR-377 was elevated versus MutmiR-377, indicating that miR-377 could directly bind with PRNCR1 (Fig. 2D). The result of RIP assay implied that compared with IgG, AGO2-bound PRNCR1 was increased (Fig. 2E), indicating that miR-377 could bind with PRNCR1.

\section{Overexpressed miR-377 inhibits breast cancerdevelopment}

Further investigation was focused on miR-377 expression in cells, and the obtained findings suggested that relative to MCF-10A, miR-377 expression in other breast cancer cells was declined in varying degrees, and MCF-7 cells had the lowest expression (Fig. 3A). Next, we introduced MCF-7 cells with mimic NC, miR377 mimic, inhibitor NC, and miR-377 inhibitor, respectively, so as to verify the transfection efficiency by RT-qPCR (Fig. 3B). The outcomes suggested that MCF-7 cells upon miR-377 mimic treatment exhibited reduced viability, retarded cell cycle progression and induced proportion of apoptosis. On the contrary, MCF-7 cells introduced with miR-377 inhibitor presented an inverse trend (Fig. 3C-E). It is suggested that miR-377 results in suppressed growth of breast cancer cells.

\section{miR-377 targets CCND2 in breast cancer}

With the aim to explore the downstream regulatory mechanism of miR-377, we screened the CCND2 gene through the online website (Fig. 4A). CCND2 is one of the cell cycle regulators, and its expression is 
elevated in many cancers. The dual luciferase reporter gene assay verified the targeting relationship between miR-377 and CCND2, and overexpressed miR-377 restricted the luciferase activity of the CCND2 reporter vector (Fig. 4B). CCND2 protein expression was reduced after miR-377 overexpression, and CCND2 protein expression was increased after miR-377 silencing (Fig. 4C, D). In the rescue experiments, mimic NC + oe-NC, miR-377 mimic + oe-NC, mimic NC + oe-CCND2 and miR-377 mimic + oe-CCND2 were introduced into MCF-7 cells, and miR-377 and CCND2 in cells was tested by RT-qPCR and western blot analysis (Fig. 4E-G). The outcomes indicated that in contrast to the mimic NC + oe-NC group, the miR-377 mimic + oe-NC group exhibited increased miR-377 expression and reduced CCND2 expression, while the mimic NC + oe-CCND2 presented elevated CCND2 expression. In addition, elevated CCND2 expression was found in the miR-377 mimic + oe-CCND2 group versus the miR-377 mimic + oe-NC group. Furthermore, in contrast to the mimic NC + oe-NC group, the miR-377 mimic + oe-NC group exhibited reduced viability, retarded cell cycle progression and induced proportion of apoptosis of MCF-7 cells. However, promoted viability, accelerated cell cycle progression and suppressed proportion of apoptosis of MCF-7 cells were found in the miR-377 mimic + oe-CCND2 group versus the miR-377 mimic + oe-NC group (Fig. 4H-J). The above results comprehensively show that miR-377 inhibits CCND2 to inhibit breast cancer cell growth.

\section{LncRNA PRNCR1/miR-377/CCND2/MEK/MAPK axis modulates breast cancer proliferation and apoptosis}

The expression of some members of the MEK/MAPK pathway (MEK1, p-MEK1, p38 MAPK, and p-p38 MAPK) in MCF-7 cells treated with oe-NC and oe-CCND2 were determined, and the results showed that overexpressed CCND2 elevated expression of p-MEK1 and p-p38 MAPK (Fig. 5A, B). Therefore, we speculated that CCND2 may regulate breast cancer proliferation and apoptosis through the MEK/MAPK pathway. Additionally, the MEK/MAPK pathway inhibitor Mirdametinib was supplemented into MCF-7 cells for detecting the expression of MEK1, p-MEK1, p38 MAPK, and p-p38 MAPK. The obtained findings suggested that suppression of the MEK/MAPK pathway (Mirdametinib) decreased the MEK1, p-MEK1, and p-p38 MAPK in MCF-7 cells (Fig. 5C, D). Furthermore, suppression of the MEK/MAPK pathway (Mirdametinib) was found to restrict viability and cell cycle entry as well as promoted apoptosis of MCF-7 cells (Fig. 5E-G). In summary, IncRNA PRNCR1 may absorb miR-377, resulting in elevated CCND2 and activated MEK/MAPK pathway, thereby leading to breast cancer growth.

\section{Discussion}

Recently, emerging evidence has supported the participation of IncRNAs in the human cancers' pathogenesis and progression [12]. To develop new cancer treatments and therapeutics, it is essential to fully understand the IncRNAs' functions and mechanisms. In this current study, we aimed to depict the mechanisms of PRNCR1 in breast cancer via miR-377/CCND2/MEK/MAPK axis.

From the obtained findings, we found that PRNCR1 was regulated in breast cancer. It is reported that increased IncRNA PRNCR1-2 expression is witnessed in breast cancer tissues, and depletion of PRNCR1-2 
suppress their proliferation rates in breast cancer cells [12]. Another study has revealed that IncRNA PRNCR1 is enhanced in breast cancer, and knockdown of PRNCR1 restricts breast cancer cells' malignant phenotypes from in vitro experiments [20]. Similarly, PRNCR1 is enhanced in colorectal cancer (CRC) tissues and PRNCR1 knockdown induces cell cycle arrest in the CRC cells [10]. Nevertheless, how PRNCR1 participates in breast cancer is barely described. Competing endogenous RNAs (ceRNAs) are capable of communicating with each other through competing for shared miRNAs [21]. From our study, we demonstrated that miR-377 expression was declined in breast cancer. miR-377 was expounded to be declined in hepatocellular carcinoma (HCC), and restoration of miR-377 suppresses HCC cell growth [22]. LncRNA PRNCR1 is a sponge in non-small cell lung cancer by competing with miR-448 [23]. Also, IncRNA PRNCR1 modulates osteogenic differentiation in osteolysis through targeting miR-211-5p [24]. However, relationship between PRNCR1 and miR-377 is barely investigated.

To probe into the molecular mechanism by which miR-377 suppressed breast cancer cell growth, we identified CCND2 as a direct target of miR-377. Aberrant expression of CCND2 has been found in testicular germ cell tumor cell lines and ovarian granulosa cell tumors [25]. In addition, it is reported that activation of CCND2 is able to induce thyroid cancer growth [26]. MiR-377 was found to participate in many tumors via different genes. Dual luciferase analysis in a study has verified that miR-377-3p could binding to HOXC6 3'-UTR region, and miR-377-3p declined HOXC6 in breast cancer [17]. miR-377 possesses the function of tumor growth suppression via decreasing Pim-3 kinase expression in order to retard pancreatic tumor growth [27]. miR-377 has also been interpreted to directly reducing ETS1 expression, which regulates the capability of renal cell carcinoma cells to migrate and invade [16]. Similarly, overexpressed miR-377 suppresses gastric cancer progression via vascular endothelial growth factor A [28]. LncRNA and mRNA integration network is of great importance in human cancers [29]. The upregulated CCND2 was evidenced to reverse the tumor inhibitory role of miR-497, further enhancing viability in breast cancer cells [19]. Nevertheless, no article has been focused on the binding relation between miR-377 and CCND2.

\section{Conclusion}

In summary, this study reports that PRNCR1 is elevated in breast cancer, and depleted PRNCR1 results in the inhibition of breast cancer progression (Fig. 6). These findings suggest that PRNCR1 may be explored

as a biomarker for breast cancer diagnostics or therapeutics. Meanwhile, our experimental data may offer a strategy for targeting miR-377/CCND2 interaction in treating breast cancer patients.

\section{Abbreviations}

miR-103a, microRNA-103a; NSCLC, non-small cell lung cancer; OTUB1, ovarian tumor domain-containing ubiquitin aldehyde binding protein 1; CSCs, cancer stem cells; DMEM, Dulbecco's modified Eagle's medium; SP, side population; oe, overexpressed; NC, negative control; MTS, 3-(4,5-dimethylthiazol-2-yl)-5(3-carboxymethoxyphenyl)-2-(4-sulfophenyl)-2H-tetr-azolium; OD, optical density; PBS, phosphate 
buffered saline; RT-qPCR, real-time quantitative PCR; GAPDH, glyceraldehyde-3-phosphate dehydrogenase; 3'UTR, 3'untranslated region; ANOVA, analysis of variation.

\section{Declarations}

\section{Acknowledgements}

Not applicable.

\section{Authors' contributions}

JOY conceived the study and participated in its design and coordination; ZLL, XBY and XC performed all experiments; YPW, LL, CPL and HL analyzed and interpreted the data; The draft was improved through discussion and editing by all the authors who read and approved the final manuscript.

\section{Funding}

Not applicable.

\section{Availability of data and materials}

All the data generated or analyzed during this study are included in this published article.

\section{Ethics approval and consent to participate}

This study was complied with all relevant ethics of human research participants, and all participants provided written informed consent.

\section{Consent for publication}

Not applicable.

\section{Competing interests}

The authors declare that they have no competing interests.

\section{References}

1. Mendes PMV, Bezerra DLC, Dos Santos LR, de Oliveira Santos R, de Sousa Melo SR, Morais JBS, et al. Magnesium in Breast Cancer: What Is Its Influence on the Progression of This Disease? Biol Trace Elem Res. 2018;184(2):334-339.

2. Perez-Garcia J, Munoz-Couselo E, Soberino J, Racca F, Cortes J. Targeting FGFR pathway in breast cancer. Breast. 2018;37126-133. 
3. DeSantis CE, Bray F, Ferlay J, Lortet-Tieulent J, Anderson BO, Jemal A. International Variation in Female Breast Cancer Incidence and Mortality Rates. Cancer Epidemiol Biomarkers Prev. 2015;24(10):1495-1506.

4. Cuchra M, Mucha B, Markiewicz L, Przybylowska-Sygut K, Pytel D, Jeziorski A, et al. The role of base excision repair in pathogenesis of breast cancer in the Polish population. Mol Carcinog. 2016;55(12):1899-1914.

5. Nazari SS, Mukherjee P. An overview of mammographic density and its association with breast cancer. Breast Cancer. 2018;25(3):259-267.

6. Monnot GC, Romero P. Rationale for immunological approaches to breast cancer therapy. Breast. 2018;37187-195.

7. Yang G, Lu X, Yuan L. LncRNA: a link between RNA and cancer. Biochim Biophys Acta. 2014;1839(11):1097-1109.

8. Chung S, Nakagawa H, Uemura M, Piao L, Ashikawa K, Hosono N, et al. Association of a novel long non-coding RNA in 8q24 with prostate cancer susceptibility. Cancer Sci. 2011;102(1):245-252.

9. Zhang A, Zhang J, Kaipainen A, Lucas JM, Yang H. Long non-coding RNA: A newly deciphered "code" in prostate cancer. Cancer Lett. 2016;375(2):323-330.

10. Yang L, Qiu M, Xu Y, Wang J, Zheng Y, Li M, et al. Upregulation of long non-coding RNA PRNCR1 in colorectal cancer promotes cell proliferation and cell cycle progression. Oncol Rep. 2016;35(1):318324.

11. Li L, Jia F, Bai P, Liang Y, Sun R, Yuan F, et al. Association between polymorphisms in long non-coding RNA PRNCR1 in 8q24 and risk of gastric cancer. Tumour Biol. 2016;37(1):299-303.

12. Pang $D, H u$ Q, Lan $X$, Lin $Y$, Duan $H$, Cao S, et al. The novel long noncoding RNA PRNCR12 is involved in breast cancer cell proliferation, migration, invasion and cell cycle progression. Mol Med Rep. 2019;19(3):1824-1832.

13. Jiang S, Miao D, Wang M, Lv J, Wang Y, Tong J. MiR-30-5p suppresses cell chemoresistance and stemness in colorectal cancer through USP22/Wnt/beta-catenin signaling axis. J Cell Mol Med. 2019;23(1):630-640.

14. Li D, Song H, Wu T, Xie D, Hu J, Zhao J, et al. MiR-519d-3p suppresses breast cancer cell growth and motility via targeting LIM domain kinase 1. Mol Cell Biochem. 2018;444(1-2):169-178.

15. Yan L, Yu MC, Gao GL, Liang HW, Zhou XY, Zhu ZT, et al. MiR-125a-5p functions as a tumour suppressor in breast cancer by downregulating BAP1. J Cell Biochem. 2018;119(11):8773-8783.

16. Wang R, Ma Y, Yu D, Zhao J, Ma P. miR-377 functions as a tumor suppressor in human clear cell renal cell carcinoma by targeting ETS1. Biomed Pharmacother. 2015;7064-71.

17. Wang X, Chen T, Zhang Y, Zhang N, Li C, Li Y, et al. Long noncoding RNA Linc00339 promotes triplenegative breast cancer progression through miR-377-3p/HOXC6 signaling pathway. J Cell Physiol. 2019;234(8):13303-13317. 
18. Zhou J, Tian Y, Li J, Lu B, Sun M, Zou Y, et al. miR-206 is down-regulated in breast cancer and inhibits cell proliferation through the up-regulation of cyclinD2. Biochem Biophys Res Commun. 2013;433(2):207-212.

19. Zhong $H$, Yang J, Zhang B, Wang X, Pei L, Zhang L, et al. LncRNA GACAT3 predicts poor prognosis and promotes cell proliferation in breast cancer through regulation of miR-497/CCND2. Cancer Biomark. 2018;22(4):787-797.

20. Guo Q, Lv S, Wang B, Li Y, Cha N, Zhao R, et al. Long non-coding RNA PRNCR1 has an oncogenic role in breast cancer. Exp Ther Med. 2019;18(6):4547-4554.

21. Qi X, Zhang DH, Wu N, Xiao JH, Wang X, Ma W. ceRNA in cancer: possible functions and clinical implications. J Med Genet. 2015;52(10):710-718.

22. Chen G, Lu L, Liu C, Shan L, Yuan D. MicroRNA-377 suppresses cell proliferation and invasion by inhibiting TIAM1 expression in hepatocellular carcinoma. PLoS One. 2015;10(3):e0117714.

23. Cheng D, Bao C, Zhang X, Lin X, Huang H, Zhao L. LncRNA PRNCR1 interacts with HEY2 to abolish miR-448-mediated growth inhibition in non-small cell lung cancer. Biomed Pharmacother. 2018;1071540-1547.

24. Gong ZM, Tang ZY, Sun XL. LncRNA PRNCR1 regulates osteogenic differentiation in osteolysis after hip replacement by targeting miR-211-5p. Biosci Rep. 2018.

25. Bouchard C, Thieke K, Maier A, Saffrich R, Hanley-Hyde J, Ansorge W, et al. Direct induction of cyclin $\mathrm{D} 2$ by Myc contributes to cell cycle progression and sequestration of p27. EMBO J. 1999;18(19):5321-5333.

26. Di W, Li Q, Shen W, Guo H, Zhao S. The long non-coding RNA HOTAIR promotes thyroid cancer cell growth, invasion and migration through the miR-1-CCND2 axis. Am J Cancer Res. 2017;7(6):12981309.

27. Chang W, Liu M, Xu J, Fu H, Zhou B, Yuan T, et al. MiR-377 inhibits the proliferation of pancreatic cancer by targeting Pim-3. Tumour Biol. 2016;37(11):14813-14824.

28. Wang CQ, Chen L, Dong CL, Song Y, Shen ZP, Shen WM, et al. MiR-377 suppresses cell proliferation and metastasis in gastric cancer via repressing the expression of VEGFA. Eur Rev Med Pharmacol Sci. 2017;21(22):5101-5111.

29. Benador IY, Veliova M, Mahdaviani K, Petcherski A, Wikstrom JD, Assali EA, et al. Mitochondria Bound to Lipid Droplets Have Unique Bioenergetics, Composition, and Dynamics that Support Lipid Droplet Expansion. Cell Metab. 2018;27(4):869-885 e866.

\section{Figures}


A

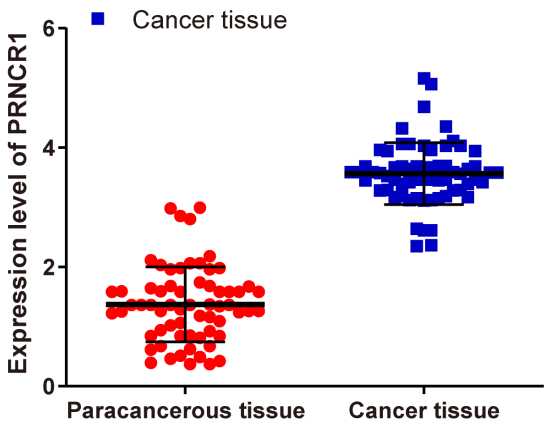

C
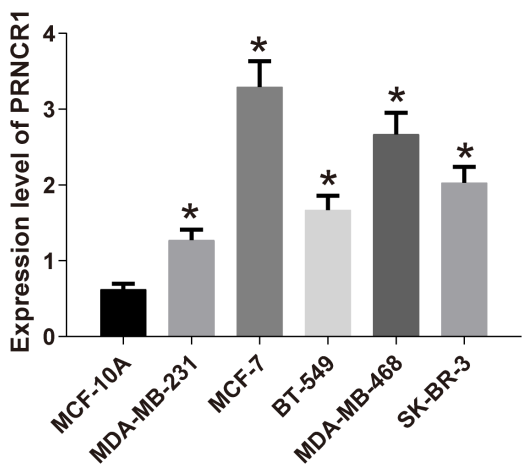

F

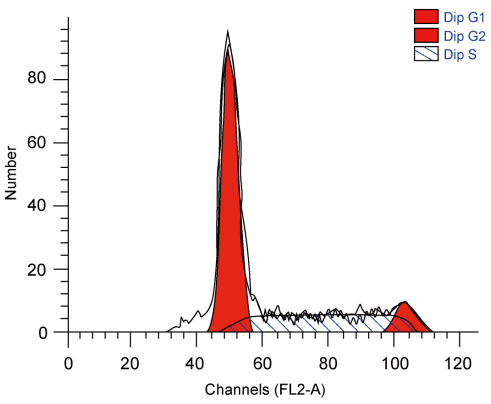

G

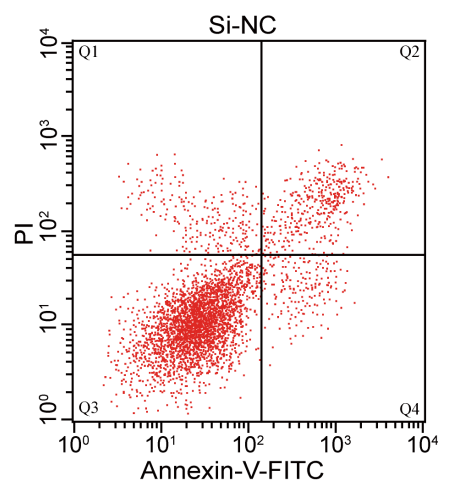

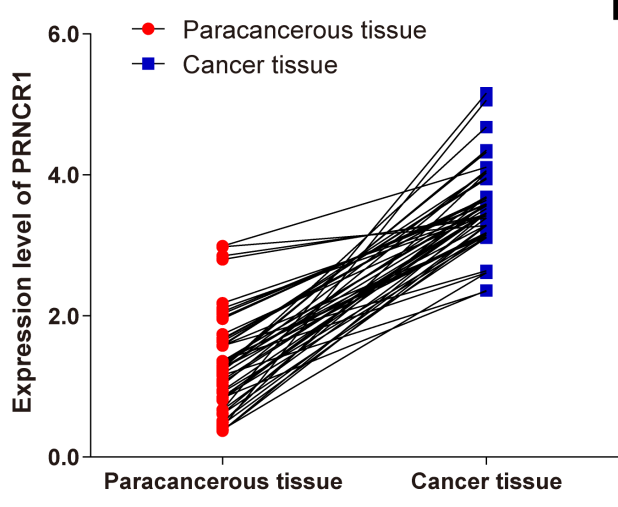

D
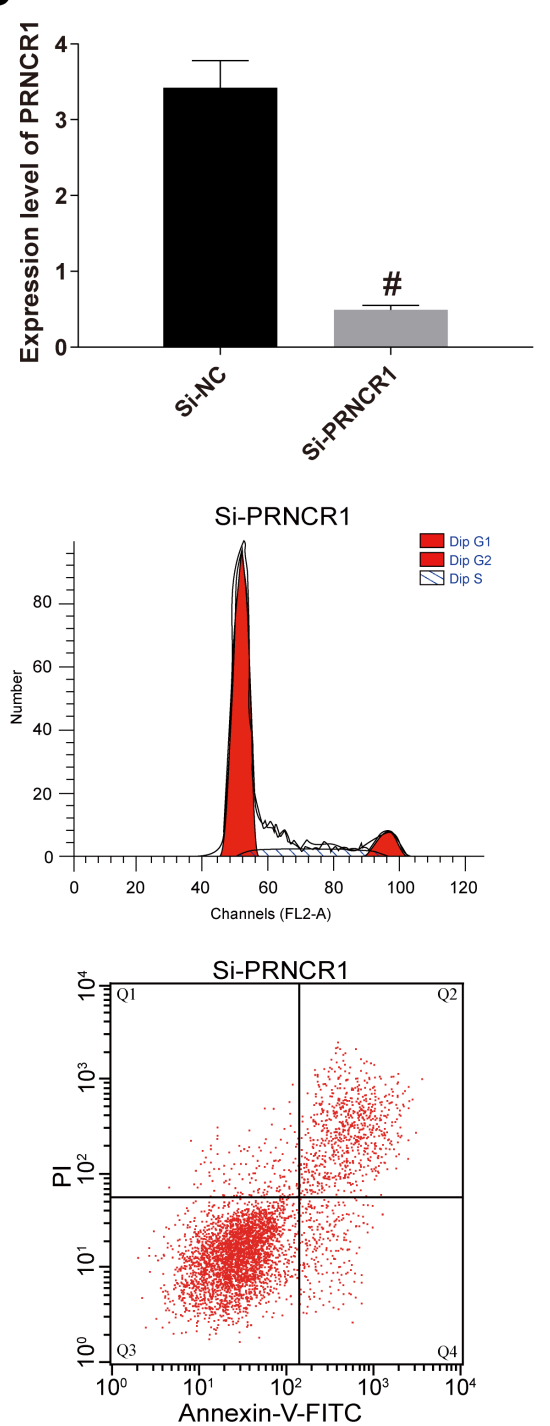

B

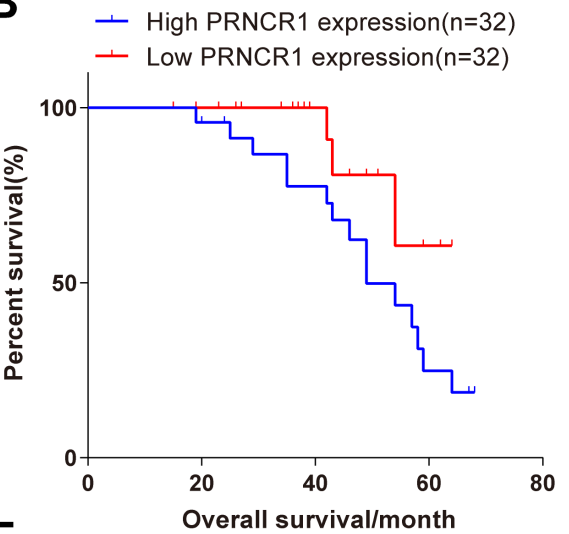

E

- Si-PRNCR1
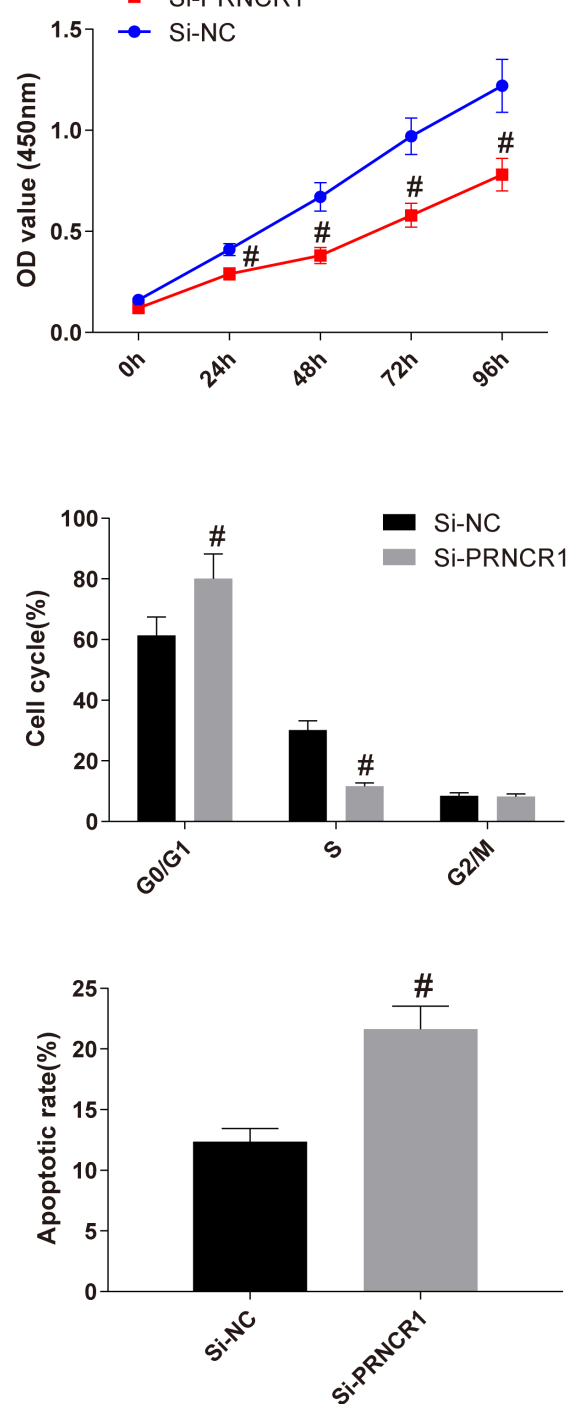

\section{Figure 1}

PRNCR1 promotes proliferation and inhibits apoptosis of breast cancer cells. A. PRNCR1 expression in breast cancer tissues and corresponding paracancerous tissues was determined by RT-qPCR. B. Relationship between PRNCR1 expression and prognosis of breast cancer patients. C. PRNCR1 expression in human breast epithelial cell line MCF-10A and breast cancer cell lines MDA-MB-231, MCF-7, BT-549, MDA-MB-468 and SK-BR-3 was determined by RT-qPCR. D. PRNCR1 expression in cells 
transfected with si-NC or si-PRNCR1. E. MCF-7 cell viability was determined by CCK-8 assay. F. Cell cycle distribution was detected by flow cytometry. G. Cell apoptosis was determined by flow cytometry. ${ }^{*} \mathrm{P}<$ 0.05 vs. MCF-10A cells; \# $P<0.05$ vs. si-NC group.

A

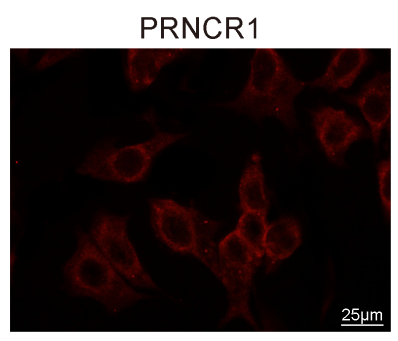

C

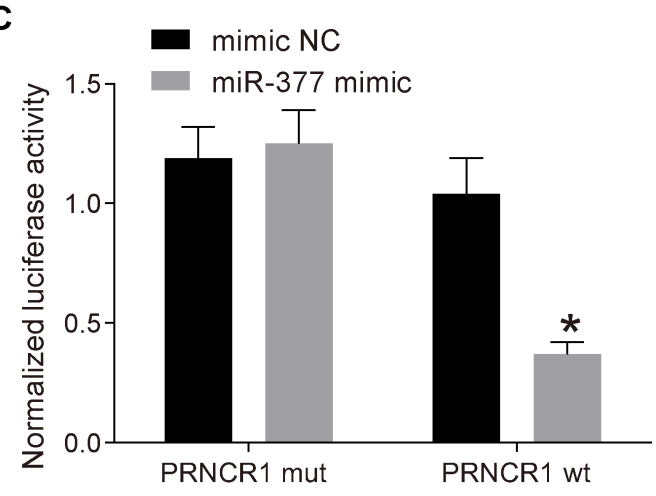

DAPI

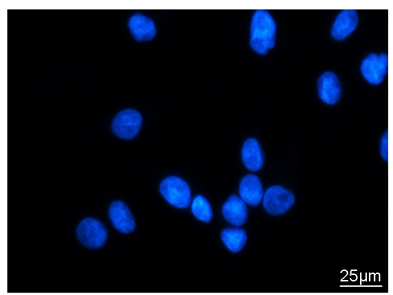

Merge

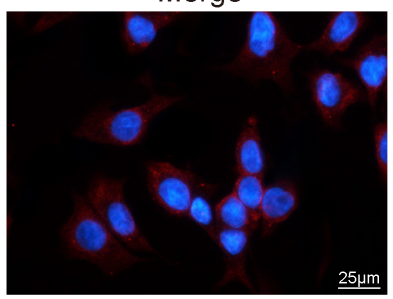

B

D

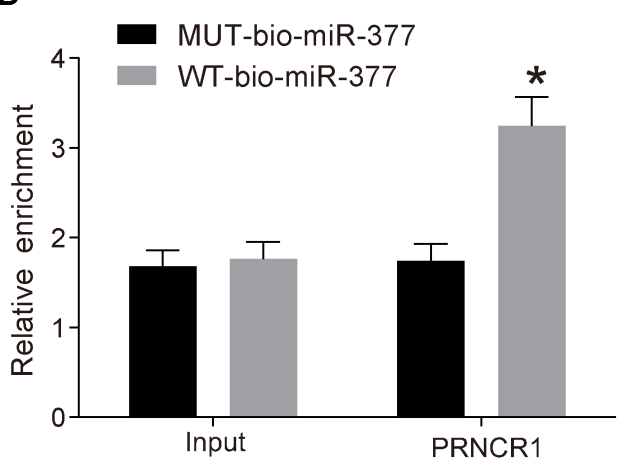

Alignment

Target: 5' aaggauuccuUCA-GUUACAUa 3

: II IIIIIII

miRNA: 3' ucaaucaccuGGUACAAUGUAu 5

E

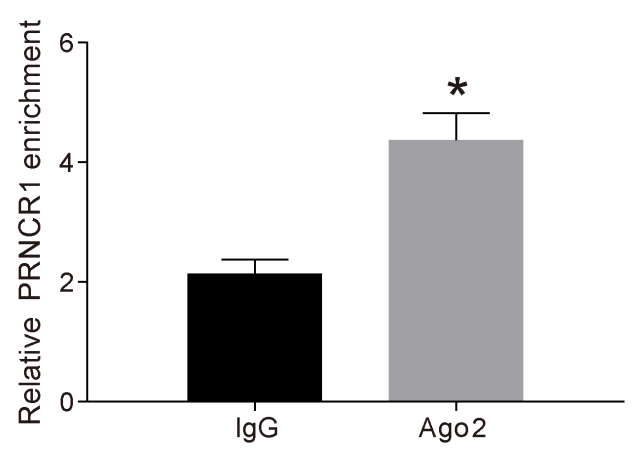

Figure 2

LncRNA PRNCR1 binds with miR-377. A. RNA-FISH detection of PRNCR1 localization in cells. B. Prediction of binding sites of PRNCR1 and miR-377. C. Dual luciferase reporter gene assay for validating the correlation between PRNCR1 and miR-377. * $P<0.05$ vs. mimic NC group. D. RNA-pull down assay detection of PRNCR1 binding to miR-377. * $\mathrm{P}<0.05$ vs. MUT-bio- miR-377 group. E. RIP assay for validating PRNCR1 function. * $\mathrm{P}<0.05$ vs. IgG group. 
A

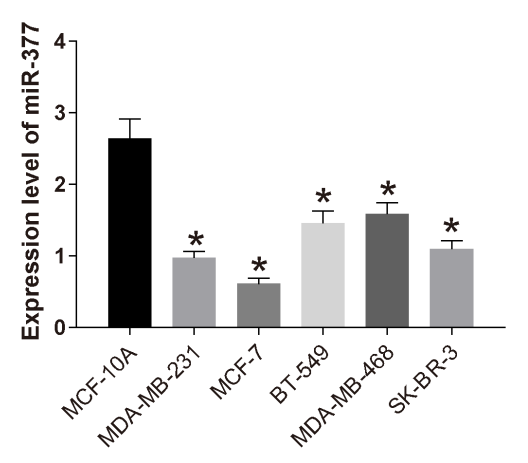

B

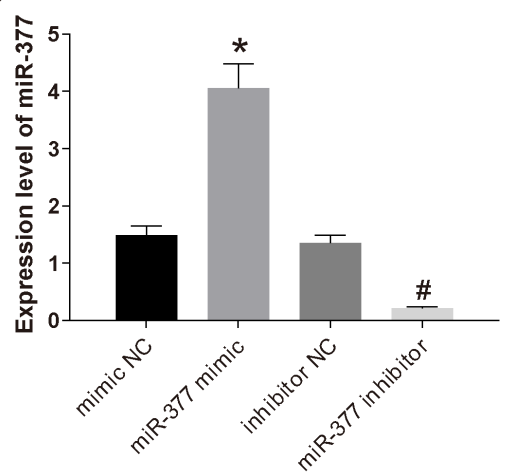

C

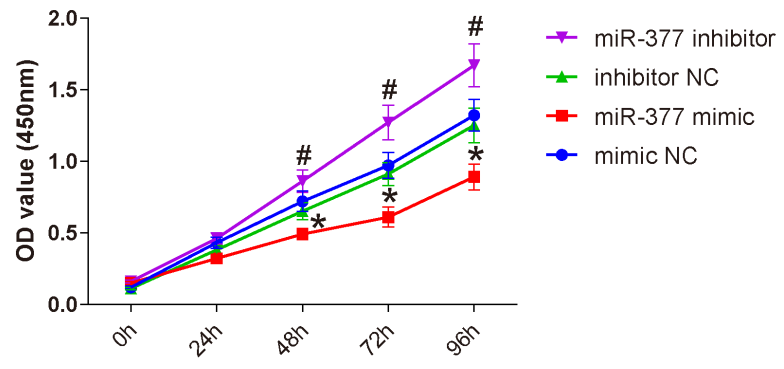

D

mimic NC

miR-377 mimic
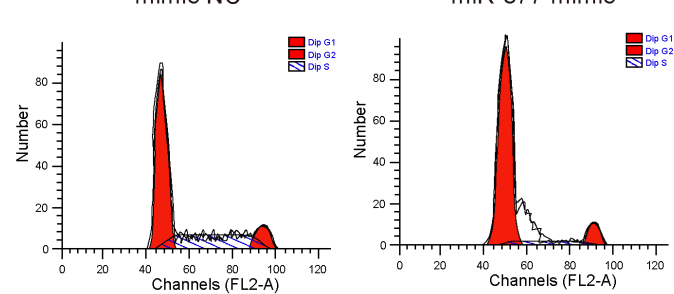

䋗:
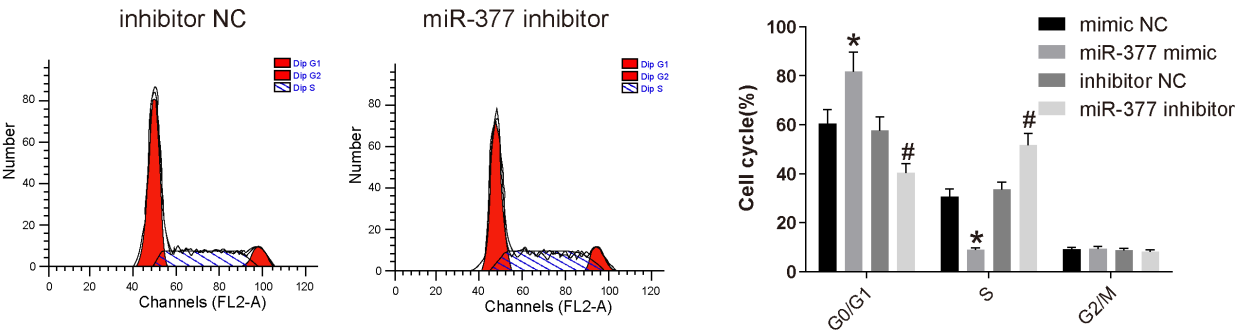

E
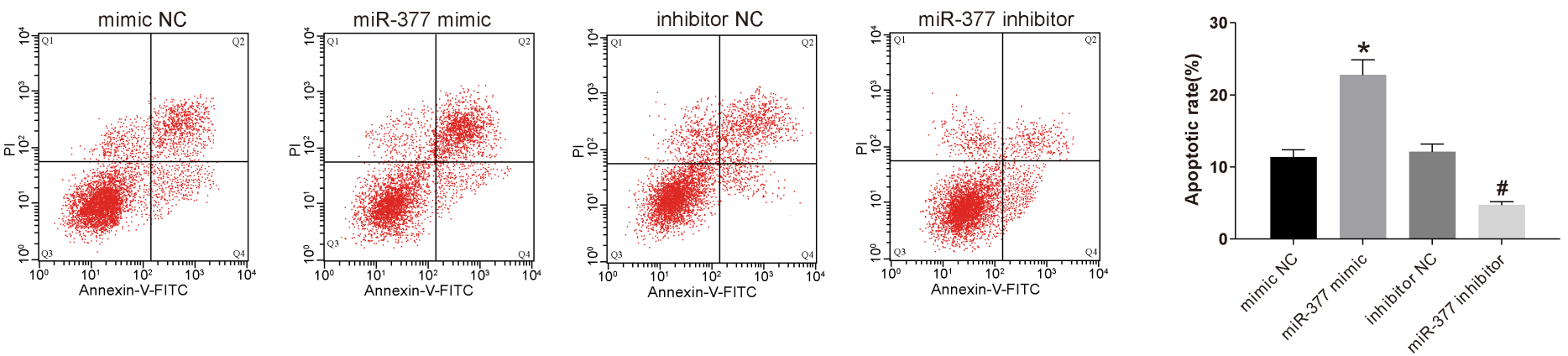

Figure 3

Overexpression of miR-377 inhibits breast cancer cell proliferation and promotes apoptosis. A. miR-377 expression in human breast epithelial cell line MCF-10A and breast cancer cell lines MDA-MB-231, MCF-7, BT-549, MDA-MB-468 and SK-BR-3 was determined by RT-qPCR. * $P<0.05$ vs. MCF-10A cells. B. miR-377 expression in cells of each group by RT-qPCR. C. MCF-7 cell viability was determined by CCK-8 assay. D. Cell cycle distribution was detected by flow cytometry. E. Cell apoptosis was determined by flow cytometry. * $\mathrm{P}<0.05$ vs. mimic NC group; \# $\mathrm{P}<0.05$ vs. inhibitor NC group. 


\section{Alignment}

Target: 5' unugcuuuaaaacaaGUGUGAu 3'

I I I I I
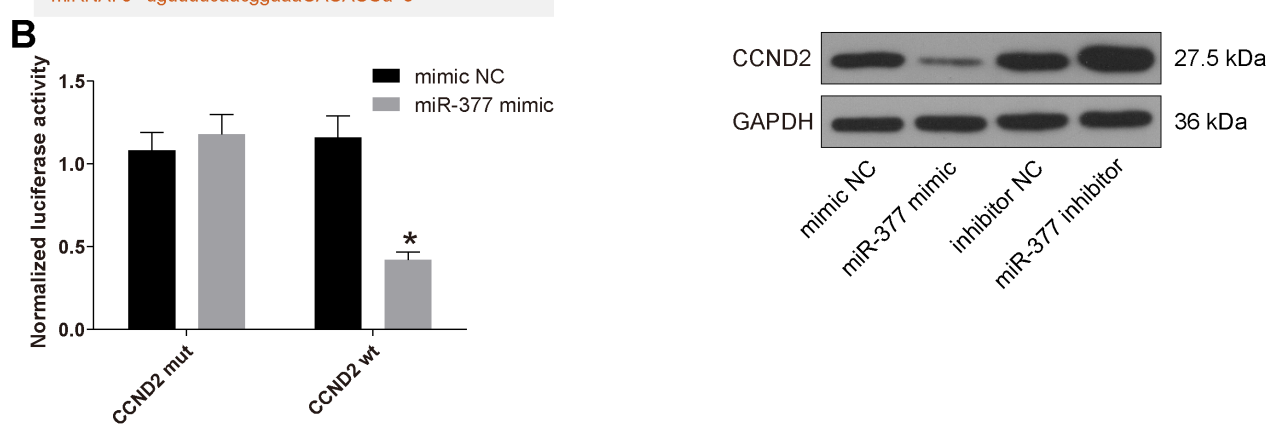

E
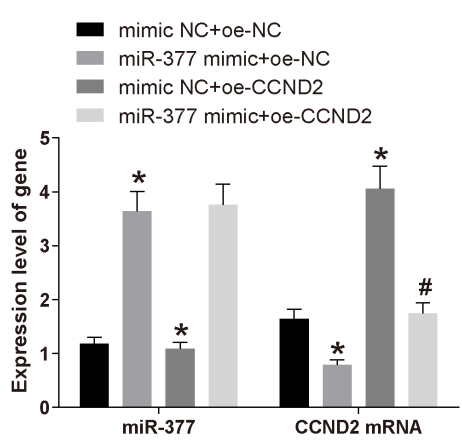

$\mathbf{F}$

G

H
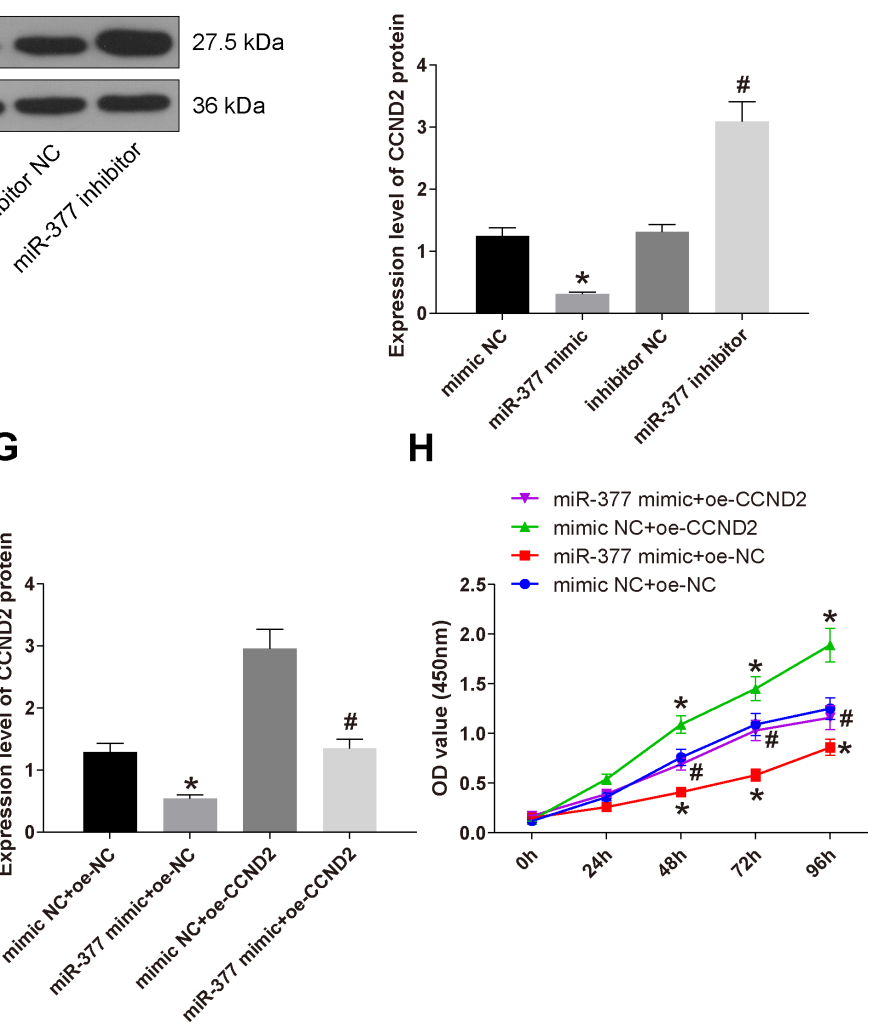

I

mimic NC+oe-NC

miR-377 mimic+oe-NC

mimic NC+oe-CCND2

miR-377 mimic+oe-CCND2

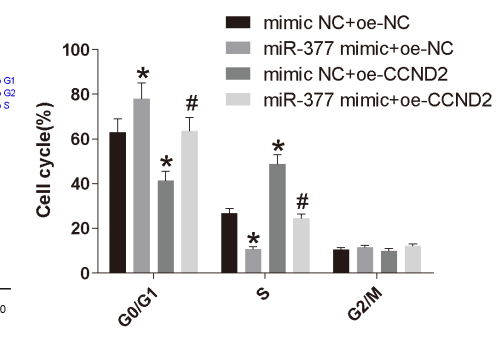

$\mathbf{J}$
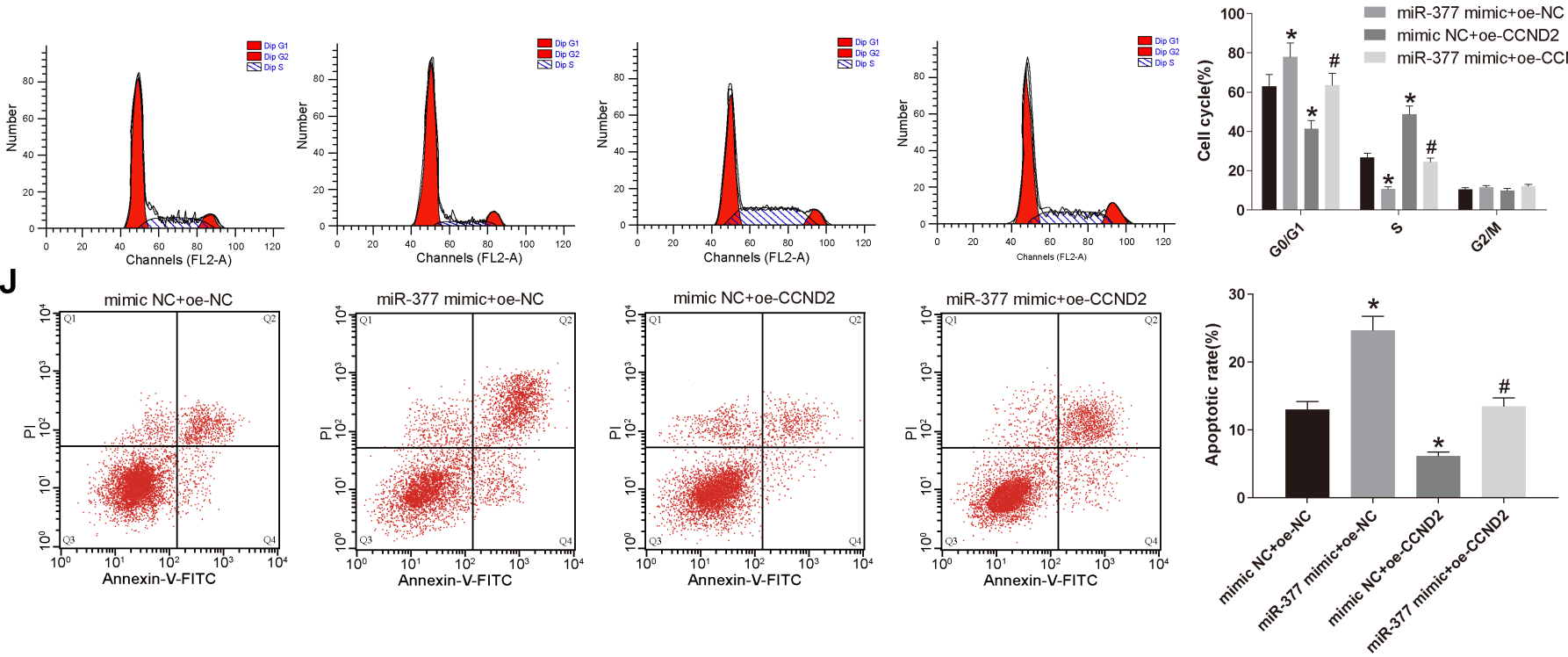

Figure 4

miR-377 targets CCND2 expression in breast cancer. A. Prediction of binding sites of miR-377 and CCND2. B. Dual luciferase reporter gene assay for validating the correlation between miR-377 and CCND2. ${ }^{*}<0.05$ vs. mimic NC group. C. Protein band of CCND2. D. Expression of CCND2 in cells of each group determined by western blot analysis. * $P<0.05$ vs. mimic NC group; \# $P<0.05$ vs. inhibitor NC group. E. Expression of miR-377 and CCND2 in cells of each group determined by RT-qPCR. F. Protein 
band of CCND2. G. Expression of CCND2 in cells of each group determined by western blot analysis. $\mathrm{H}$. MCF-7 cell viability was determined by CCK-8 assay. I. Cell cycle distribution was detected by flow cytometry. J. Cell apoptosis was determined by flow cytometry. ${ }^{*} \mathrm{P}<0.05$ vs. mimic NC + oe-NC group; \# $\mathrm{P}<0.05$ vs. miR-377 mimic + oe-NC group.

A
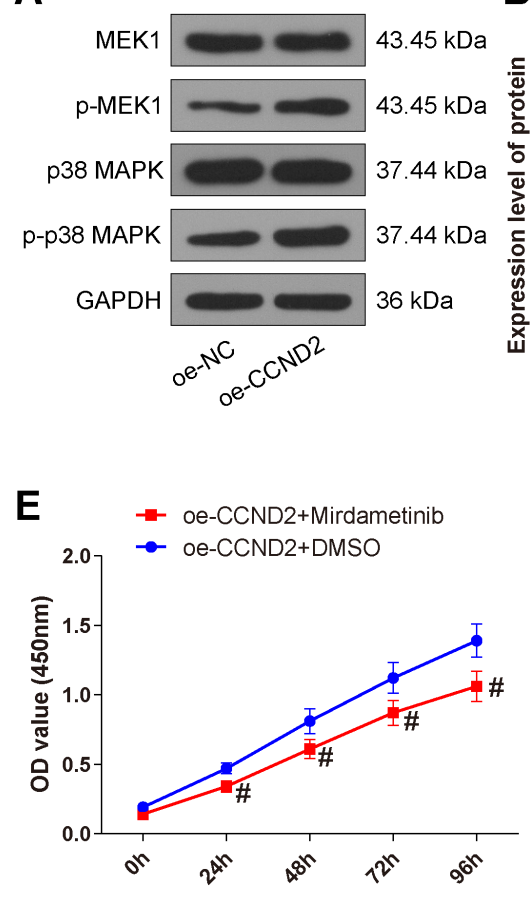

G

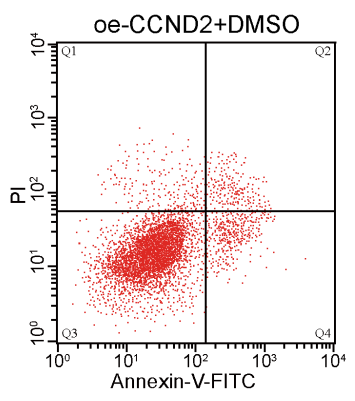

B

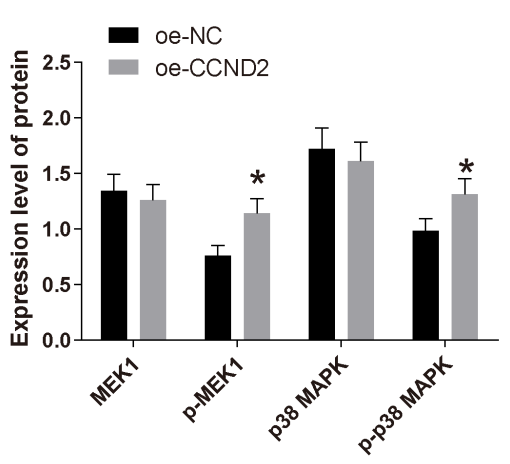

$\mathbf{F}$

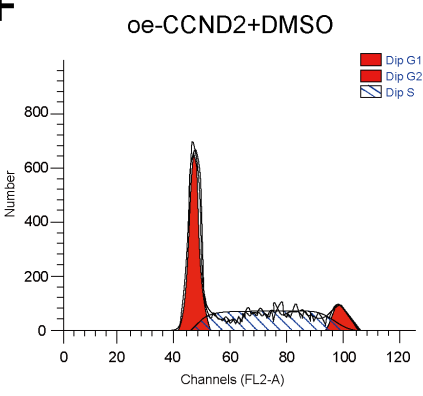

C

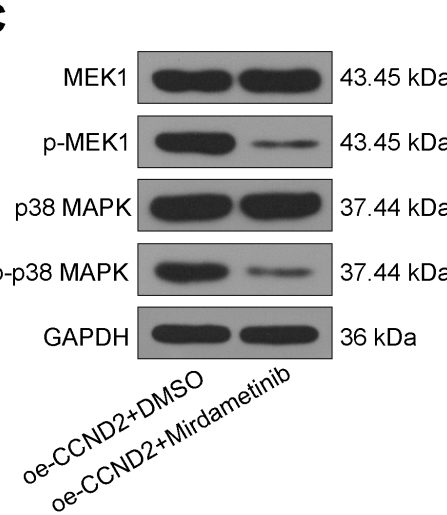

D

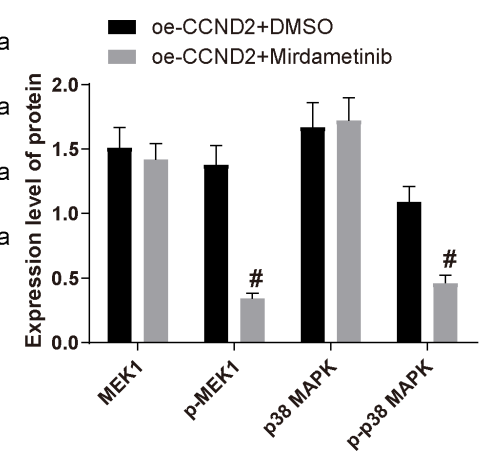

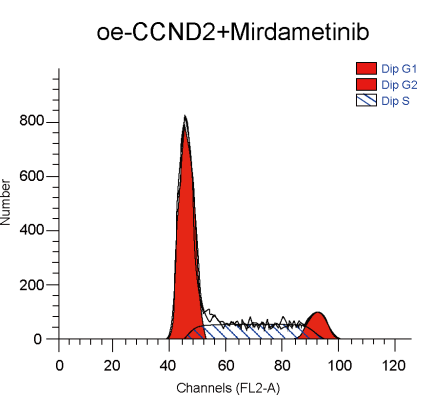

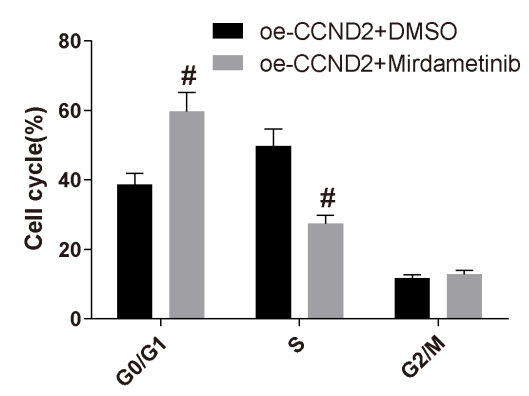

Figure 5

CCND2 activates MEK/MAPK pathway. A. Protein bands of MEK1, p-MEK1, p38 MAPK, and p-p38 MAPK. B. MEK1, p-MEK1, p38 MAPK, and p-p38 MAPK expression levels in cells treated with oe-NC or oe-CCND2 by western blot analysis. C. Protein bands of MEK1, p-MEK1, p38 MAPK, and p-p38 MAPK. D. MEK1, pMEK1, p38 MAPK, and p-p38 MAPK expression levels in cells by western blot analysis. E. MCF-7 cell viability was determined by CCK-8 assay. F. Cell cycle distribution was detected by flow cytometry. G. Cell apoptosis was determined by flow cytometry. ${ }^{*} \mathrm{P}<0.05$ vs. oe-NC group; $\# \mathrm{P}<0.05$ vs. oe-CCND2 + DMSO group. 


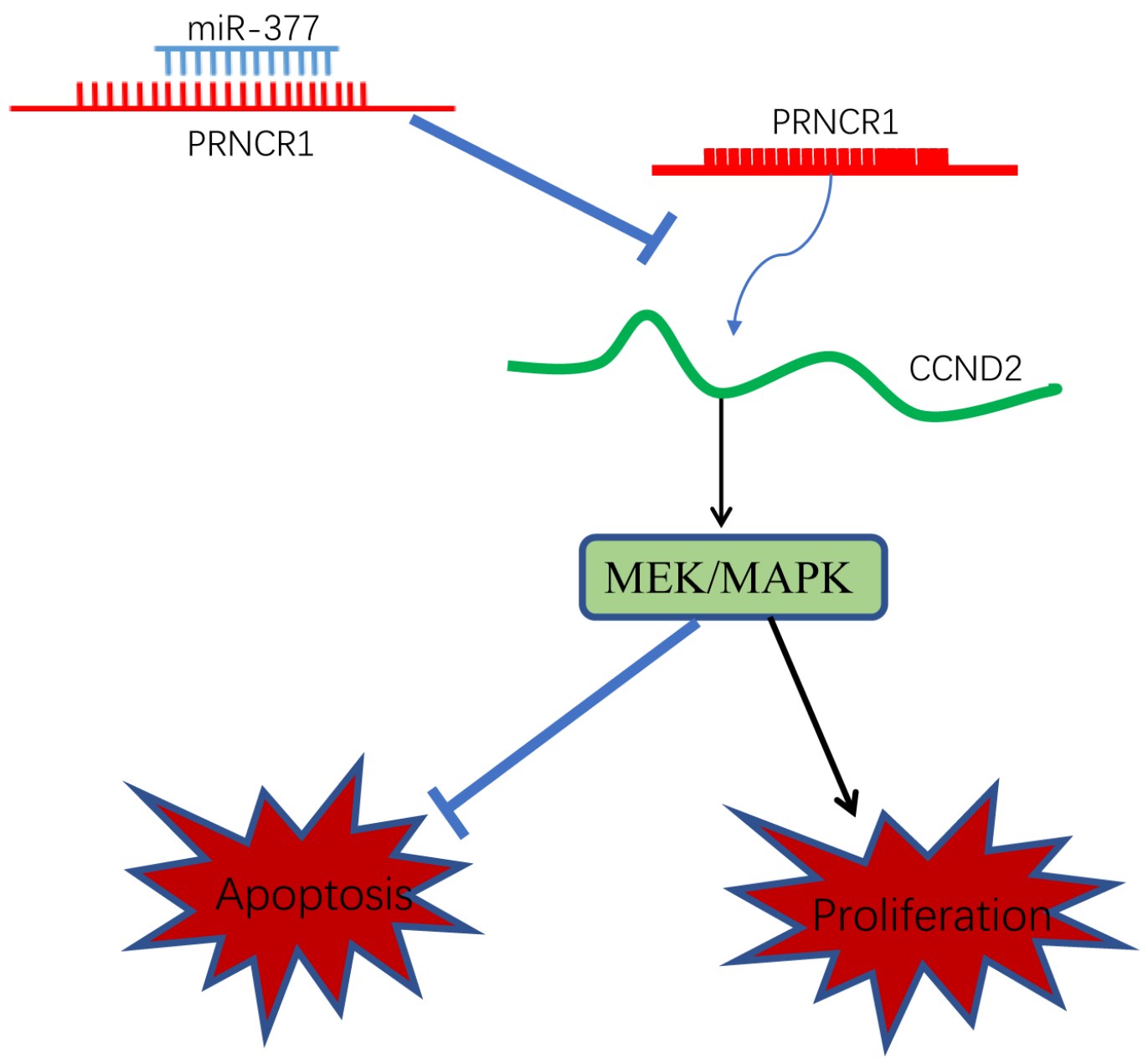

Figure 6

The mechanistic diagram depicts that IncRNA PRNCR1 may competitively bind to miR-377, leading to upregulation of CCND2 expression, which in turn leads to activation of the MEK/MAPK pathway, thereby promoting breast cancer cell proliferation and inhibiting apoptosis. 